\title{
3-D Eddy Current and Fringing-Flux Distribution in an Axial-Flux Permanent-Magnet Synchronous Machine With Stator in Laminated Iron or SMC
}

\author{
Bart Scheerlinck ${ }^{1,2}$, Herbert De Gersem ${ }^{3}$, and Peter Sergeant ${ }^{1,2}$ \\ ${ }^{1}$ Electrical Energy Laboratory, Department of Electrical Energy, Systems and Automation, \\ Ghent University, Ghent 9000, Belgium \\ ${ }^{2}$ Electrical Energy Research Group, Department of Industrial Technology and Construction, \\ Ghent University, Ghent 9000, Belgium \\ ${ }^{3}$ Institut für Theorie Elektromagnetischer Felder, Technische Universität Darmstadt, \\ Darmstadt 64289, Germany
}

This paper compares the iron losses generated by the concentrated excitation windings of an axial-flux permanent-magnet synchronous machine (AFPMSM) in the stator core elements constructed with laminated silicon steel sheets (LSSS) or with soft magnetic composite (SMC). As the flux mainly flows in one direction, we use grain-oriented LSSS. In order to provide a very accurate comparison, individual laminations are modeled in the 3-D finite-element model, including the coating layer. The model accounts for the magnetic stray field (fringing field) that causes eddy currents in the plane of the sheets, in addition to the main flux causing the classical eddy-current loss. The losses caused by the 3-D eddy-current pattern, as well as hysteresis and excess losses, are computed and measured for a test setup. A comparison of losses is made for the SMC mounted in the same setup. SMC does not suffer from eddy currents due to fringing flux. Although SMC has a 16 times higher loss than the LSSS at $1 \mathrm{~T}$ and $50 \mathrm{~Hz}$ as measured with an Epstein frame, it was shown that the iron losses in the SMC in the AFPMSM setup are only 1.5 times higher than for the LSSS.

Index Terms - Eddy currents, iron losses, stray fields.

\section{INTRODUCTION}

$\mathbf{T}$ HE stator of a yokeless and segmented armature axial-flux permanent-magnet synchronous machine (AFPMSM) is built of individual core elements and excited by concentrated windings. Nowadays, these core elements are mostly constructed of laminated silicon steel sheets (LSSS). Two types of relevant eddy-current effects occur [1]. The first type is due to the main magnetic field parallel to the laminations, which induces narrow current loops within each individual sheet. The second type comes from the magnetic stray field, also called the fringing field, which has components perpendicular to the laminations, and induces wide current loops parallel to the sheets. The use of grain-oriented silicon steel is appropriate for the AFPMSM stator core elements, because the main flux follows the rolling direction. A stack of grain-oriented LSSS is both pseudoanisotropic and crystalline anisotropic. The pseudoanisotropy comes from the heterogeneity caused by the insulation between adjacent sheets. The crystalline anisotropy is due to the manufacturing (rolling) process of the individual sheets. As an alternative to LSSS, soft magnetic composite (SMC) can carry 3-D flux paths, and may in some applications increase the torque/weight ratio of the machine. SMC is mainly used in the machines with a complex 3-D path such as transverse flux machine [2] or axial-flux machines [3]. On the one hand, the isotropic behavior of the SMC is an advantage for these types of machines. Moreover, SMC does not suffer from induced currents due to fringing

Manuscript received March 20, 2015; revised June 5, 2015; accepted June 6, 2015. Date of publication June 16, 2015; date of current version October 22, 2015. Corresponding author: B. Scheerlinck (e-mail: bart.scheerlinck@ugent.be).

Color versions of one or more of the figures in this paper are available online at http://ieeexplore.ieee.org.

Digital Object Identifier 10.1109/TMAG.2015.2443555

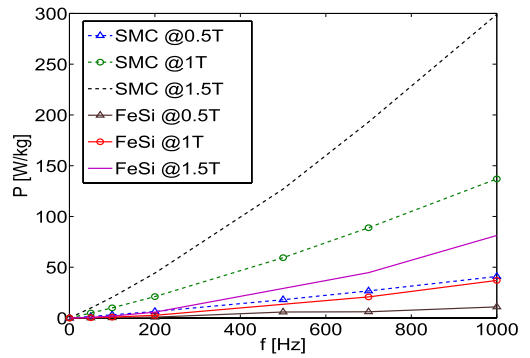

Fig. 1. Losses for Somaloy prototype SMC and FeSi on an Epstein frame for $0.5,1$, and $1.5 \mathrm{~T}$.

field perpendicular to the lamination. On the other hand, SMC has usually a lower magnetic permeability and higher losses in the frequency range that is relevant in the electrical machines. This paper compares both materials including a detailed study of the eddy-current phenomena in the laminations.

\section{Simplified GeOMETRY FOR AN ACCURATE IRON LOSS STUDY OF AFPMSM}

The machine under study is an axial-flux permanent-magnet machine with dual rotor and 15 teeth (see Fig. 3). In order to provide a very accurate study of the iron loss, a simplified setup of this machine without any rotating parts is constructed. This eliminates uncertainties on air-gap width, as well as bearing and winding losses. Fig. 4(a) shows the geometry of the simplified setup, consisting of only two laminated or SMC stator teeth of the AFPMSM and two back iron plates closing the magnetic circuit. The teeth have concentrated tooth-coil windings. A transient 3-D finite-element model (FEM) is created for this simplified setup, in order to allow a detailed analysis of the 3-D eddy-current patterns in the individual laminations. The middle part of the stack is homogenized, 


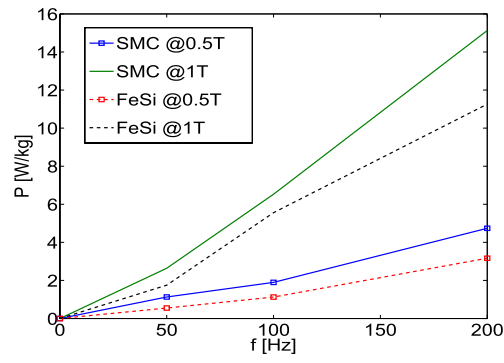

Fig. 2. Losses for Somaloy prototype SMC and FeSi on the AFPMSM setup at 0.5 and $1 \mathrm{~T}$.

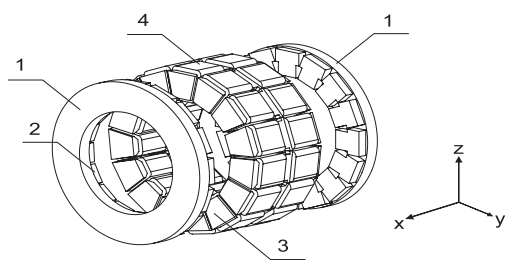

Fig. 3. AFPMSM. 1: rotor disk. 2: permanent magnet. 3: laminated stack 4: winding [4].

with the homogenization technique of [5]. There, the losses are computed using a 1-D diffusion model [6]. The FE model and the 1-D diffusion model are described in Section III.

The magnetic material model is based on the Epstein frame measurements. The Epstein frame does not contain any air gaps; therefore, the amount of fringing flux is negligible in this setup. For the laminated sheets, the classical losses, including in plane eddy-current losses, are computed by the transient 3-D model. Hysteresis and excess losses are calculated using a loss model fitted on the basis of the Epstein frame measurements and a 1-D diffusion model of the lamination. For SMC, the classical loss is computed via a homogenized method in FEM. The hysteresis loss is computed based on the Epstein frame measurements. Details about the material characterization are given in Section IV. Fig. 1 shows the losses for SMC and FeSi on an Epstein frame for 0.5, 1, and $1.5 \mathrm{~T}$.

\section{NUMERICAL MODEL}

\section{A. Finite-Element Model in the Case of Laminated Stack}

Symmetries allow to only model one eighth of the geometry by selecting appropriate boundary conditions. Unlike many approaches, where the lamination stack is modeled as a bulk material, this paper models a number of laminations explicitly, including the thin insulating coatings in a part of the geometry [Fig. 4(b)]. The remaining part of the stack is homogenized.

Eddy currents caused by stray fields need to be modeled with a high accuracy. This is a challenge for the numerical model for three reasons. The first reason is the geometric scaling problem: the large geometrical disproportion between the lamination and insulation thicknesses and the device size. The second reason is that the maximal mesh size must be sufficiently smaller than the skin depth in each individual lamination. The third reason is that eddy currents due to stray fields are a secondary effect and are therefore small compared with the excitation currents. As a consequence, the solution for the eddy currents may be inaccurate, even when the magnetic field converged [7], [8]. The field model is based on a 3-D nonlinear time-dependent magnetoquasistatic field formulation.

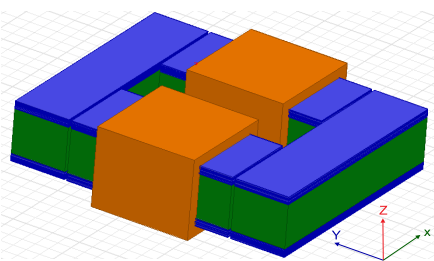

(a)

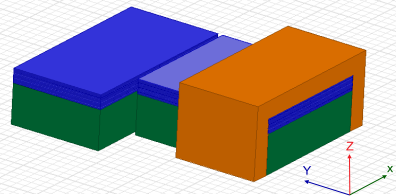

(b)
Fig. 4. (a) Simplified setup and (b) FEM geometry exploiting symmetry and showing explicitly modeled laminations (blue area) and the homogenized part of the lamination stack (green area).

The second-order tetrahedral finite elements are used together with the $T-\Omega$ formulation [9], which uses an additional current vector potential $\vec{T}$ in the eddy-current region $\Omega_{c}$ and a reduced magnetic scalar potential $\phi$ in the whole problem region $\Omega$. The current density $\vec{J}$ and the magnetic field $\vec{H}$ are calculated from the potentials as

$$
\begin{aligned}
& \vec{J}=\vec{\nabla} \times \vec{T} ; \quad \vec{H}=\vec{T}_{0}+\vec{T}-\vec{\nabla} \phi \text { in } \Omega c \\
& \vec{J}=\vec{\nabla} \times \vec{T}_{0} ; \quad \vec{H}=\vec{T}_{0}-\vec{\nabla} \phi \text { in } \Omega-\Omega c
\end{aligned}
$$

where $\vec{T}_{0}$ is the impressed current vector potential.

\section{B. Lamination Stack: Individually Modeled Sheets}

For the core elements made from LSSS, even for low excitation currents, the outer sheets of an LSSS stack saturate very quickly [see Fig. 9(b)]. This is mainly caused by the stray fields perpendicular to the lamination. As a consequence, the eddy currents induced by these stray fields will be the highest in the first few sheets close to the excitation winding. To accurately compute the $\Omega_{c}$ eddy currents caused by the stray fields, the first few $0.23 \mathrm{~mm}$-thick sheets closest to the excitation winding are explicitly resolved by the FE mesh by defining them in the FE model as individual domains, separated by the thin insulating (coating) layers of $15 \mu \mathrm{m}$. The two types of eddy currents as described in Section I cannot be strictly separated in the non-linear case, because they influence each other. For this reason, both eddy currents are calculated together in the first few sheets closest to the excitation winding. Knowing that the magnetic induction is always enforced to be in the rolling direction, it is acceptable to neglect the crystalline anisotropy and to use a scalar magnetic material model.

\section{Lamination Stack: Homogenization in the Center Part of the Stator Stack}

The remaining center part of the laminated stack is modeled as a homogenized bulk material with anisotropic magnetic and electric properties, as suggested in [10]. The permeability in the direction perpendicular to the stack is determined by the coating thickness and the lamination thickness. The pseudoanisotropy is considered by use of an anisotropic electrical conductivity. The classical losses in this homogenized center part are calculated separately using a 1-D finite difference diffusion model of half the lamination thickness.

\section{1-D Finite Difference Diffusion Model for the}

\section{Classical Losses in the Laminations}

For calculating the classical losses, very accurately and with a high spatial accuracy, Bertotti's low-frequency approximation is no longer an option in the considered grain-oriented 


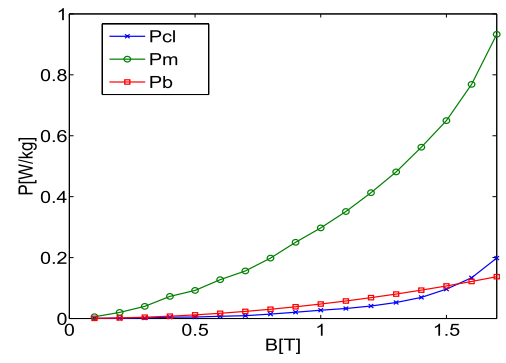

Fig. 5. Comparison of the classical losses $\left(P_{\mathrm{cl}}\right)$ calculated with the 1-D diffusion model of Section III-D, the classical losses calculated with the low-frequency approximation of Bertotti $\left(P_{b}\right)$, and the total measured losses $\left(P_{m}\right)$.

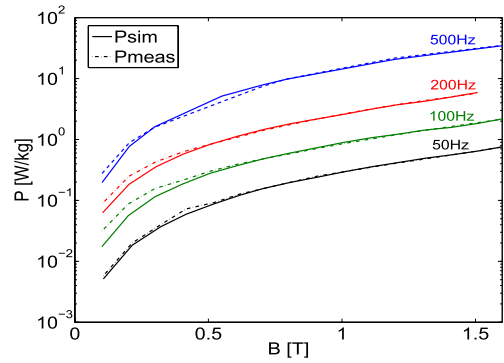

Fig. 6. Fitted total iron losses [ $P_{\text {sim }}$ in the rolling direction of M100-23P grain-oriented silicon steel based on the Epstein frame measurements $\left.\left(P_{\text {meas }}\right)\right]$.

material with very high relative permeability (up to 40000), because of skin effects, even for low frequencies. For this reason, a 1-D space diffusion problem is solved for half of the lamination thickness. The magnetic field intensity in the out-of-plane direction is calculated from

$$
\frac{\partial^{2} H}{\partial x^{2}}=\sigma \frac{d B}{d H} \frac{\partial H}{\partial t}
$$

where $H$ and $B$ are, respectively, the magnetic field and magnetic induction component in the out-of-plane direction, $\sigma$ is the electrical conductivity, and $x$ is the transverse direction of the lamination. Using the explicit equation for calculating the magnetic field at each node, the following restriction arises to ensure numerical stability:

$$
\frac{P}{\beta h^{2}} \leq \frac{1}{2}
$$

where $\beta=\sigma d B / d H, P$ is the time interval, and $h$ is the mesh length [11]. To ensure stability, it becomes advantageous to apply the magnetic field strength instead of the magnetic induction in function of time. A sinusoidal excitation gives a non-sinusoidal response in the non-linear case. For this reason, the non-sinusoidal field intensities measured with the Epstein frame are used for exciting the 1-D model.

\section{E. SMC}

For the core elements made of SMC, the classical losses are directly calculated from the 3-D non-linear FE model, as described in Section IV. Evidently, no laminations need to be modeled here. For both materials, single-valued $B H$-curves, measured on the Epstein frames, are used.

\section{Material Modeling}

The total iron losses are measured with the Epstein frame for frequencies up to $1000 \mathrm{~Hz}$ and for flux densities up to $1.8 \mathrm{~T}$ for the grain-oriented silicon steel, and up to $1.4 \mathrm{~T}$ for SMC.

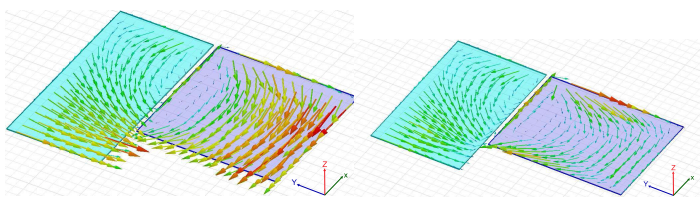

(a)

(b)

Fig. 7. (a) Induced eddy-current field in top sheet of LSSS stack [see Fig. 4(b)], at point $1.57 \mathrm{~ms}$, where the eddy currents due to the main flux are dominant. (b) Induced eddy-current field in top sheet of LSSS stack [see Fig. 4(b)], at point $1.72 \mathrm{~ms}$ where the eddy currents due to stray fields are dominant.

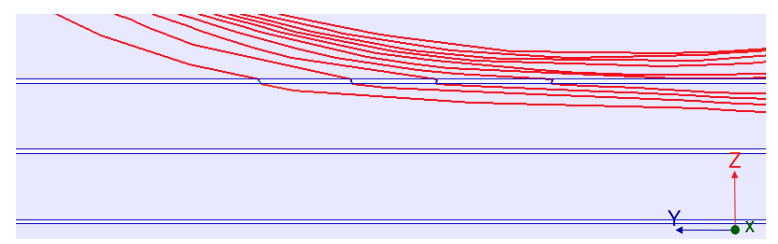

Fig. 8. Fringing fieldlines in the outer sheets of Fig. 4(b).

Because the skin effect is substantial, especially at the higher frequencies, in spite of the thin lamination thickness of $0.23 \mathrm{~mm}$, the 1-D diffusion model from Section III-D is used to compute the classical loss. Fig. 5 shows the classical losses calculated with the 1-D diffusion model at $50 \mathrm{~Hz}$, together with the total losses and the classical losses calculated with the low-frequency approximation of Bertotti. The hysteresis and excess losses are calculated by the Bertotti formulas [12] fitted on the basis of the Epstein frame measurements

$$
\begin{aligned}
& P_{h}=a B^{\alpha} f \\
& P_{e}=c B f(\sqrt{1+d B f}-1)
\end{aligned}
$$

where $P_{h}$ and $P_{e}$ are the hysteresis and the excess losses, respectively, and $a, \alpha, c$, and $d$ are four material specific coefficients.

In the numerical model, the classical loss is computed using the 3-D transient FE model in the individually modeled laminations and by the 1-D diffusion model in the homogenized part. The hysteresis and excess losses are added a posteriori based on the above fitted coefficients. The fitted losses in the rolling direction based on the Epstein frame measurements for M100-23P grain-oriented silicon steel are plotted in Fig. 6 .

\section{Detailed Analysis of Eddy CURRents IN LSSS}

The first type of eddy currents-eddy currents due to the main flux - are resistance limited. The second type of eddy currents - the eddy currents caused by stray fields - are mostly limited by their own field and are said to be inductance limited. Knowing the latter effect, a phase shift in time between the two types of eddy currents is expected. For a calculation at a frequency of $333 \mathrm{~Hz}$, it can be seen in Fig. 7(a) that at $1.57 \mathrm{~ms}$, when the change in main flux is maximum, the classical eddy currents are dominant. It can be seen in Fig. 7(b) that later in time, at $1.72 \mathrm{~ms}$, when the change in stray flux is maximum, that the eddy currents due to stray fields are dominant. The fringing field causes flux redistribution in the individual sheets due to saturation. This redistribution causes even more perpendicular flux falling into the adjacent sheets, as shown in Fig. 8. The instantaneous stray field losses in the individual top sheets, with the sheet numbering starting 


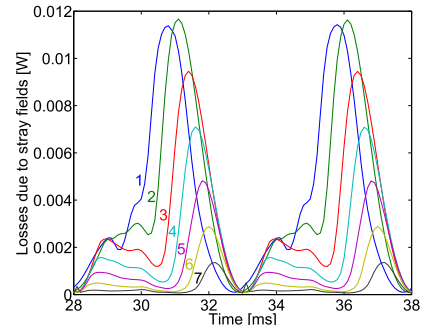

(a)

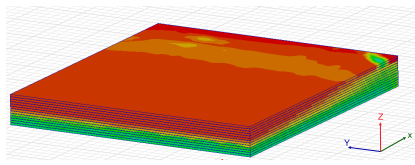

(b)
Fig. 9. (a) Instantaneous losses due to stray fields in function of time in the individual top sheets, with the sheet numbering starting from the top of the LSSS stack [see Fig. 4(b)]. (b) Magnetic induction in the top sheets of Fig. 4(b), ranging from light green $0.7 \mathrm{~T}$ to red $2 \mathrm{~T}$.

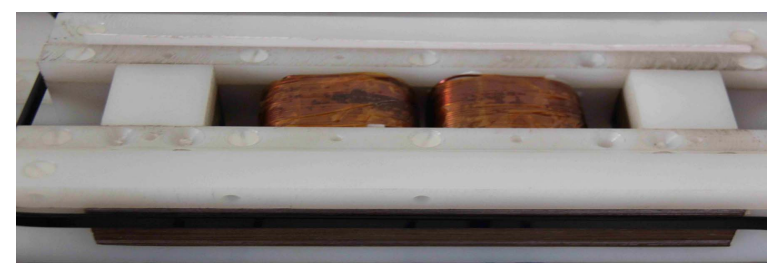

Fig. 10. Dedicated AFPMSM test setup.

from the top of the LSSS stack of Fig. 4(b), are shown in Fig. 9(a). As can be seen, there is a clear time shift between the individual sheet losses, due to the shielding effect of the individual sheets, which delays the penetrating fringing field in time.

\section{DedicAted EXPERIMENTAL SETUP}

In order to evaluate the FEM results, a rigid dedicated experimental setup without moving parts is built, as shown in Fig. 10. The air gap can be varied, by putting a number of polyamide sheets with a thickness of $1 \mathrm{~mm}$ between the stator teeth and the rotor back iron. For each material, four stacks are positioned at a pole width of $50 \mathrm{~mm}$ from each other. The yoke with a thickness of $20 \mathrm{~mm}$ is larger than the real back iron of a permanent-magnet synchronous motor, in order to keep the losses of the back iron low compared with those in the lamination stack of the motor teeth. All LSSS are cut with a water jet, in order to have minimal degradation of magnetic properties. Besides the copper, lamination stacks, and/or SMC teeth, all surrounding materials are polyamid.

\section{RESULTS}

Fig. 2 shows the loss measurements on the AFPMSM setup for the SMC and the FeSi stator core elements at 0.5 and $1 \mathrm{~T}$. The difference between loss measurement results for the AFPMSM for the motor teeth made of FeSi or SMC is small because of the 3-D flux path capability of SMC and the losses in the back iron. The 3-D model is evaluated and compared with the measurement data for the two types of materials. Fig. 11 shows the total measured and total modeled loss of the AFPMSM setup for LSSS at frequencies of 50, 100, and $200 \mathrm{~Hz}$. It can be seen that the loss due to fringing field increases more rapidly than the total loss, because the loss due to fringing field is the largest loss component depending

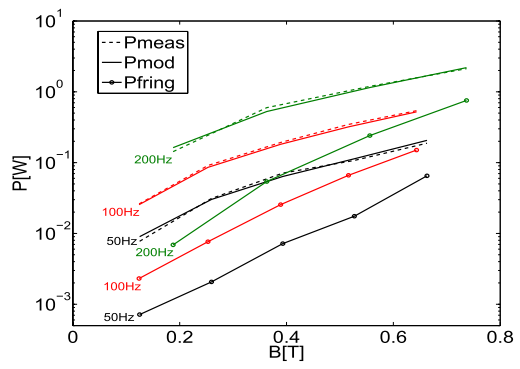

Fig. 11. Total measured losses $\left(P_{\text {meas }}\right)$, total modeled losses $\left(P_{\text {mod }}\right)$, and losses due to fringing field ( $P_{\text {fring }}$ ) of the AFPMSM setup for LSSS at frequencies of 50,100, and $200 \mathrm{~Hz}$.

on the square of the magnetic induction. The highest increase of total loss due to fringing flux is $\sim 34 \%$. This value will even increase when the air gap increases.

\section{CONCLUSION}

When measured with an Epstein frame, the losses in the SMC are $16 \times$ larger than the losses in the LSSS. However, when mounted as a stator core element in an AFPMSM, this difference is lowered to a factor 1.5. This fact is explained by the substantial eddy-current losses induced in the LSSS by fringing fluxes perpendicular to the laminates. The non-linear transient 3-D model clearly indicates the influence of saturation and the time patterns of the losses in the outer lamination sheets.

\section{REFERENCES}

[1] H. De Gersem, S. Vanaverbeke, and G. Samaey, "Three-dimensionaltwo-dimensional coupled model for eddy currents in laminated iron cores," IEEE Trans. Magn., vol. 48, no. 2, pp. 815-818, Feb. 2012.

[2] A. Schoppa and P. Delarbre, "Soft magnetic powder composites and potential applications in modern electric machines and devices," IEEE Trans. Magn., vol. 50, no. 4, Apr. 2014, Art. ID 2004304.

[3] O. Maloberti et al., "3-D-2-D dynamic magnetic modeling of an axial flux permanent magnet motor with soft magnetic composites for hybrid electric vehicles," IEEE Trans. Magn., vol. 50, no. 6, Jun. 2014, Art. ID 8201511.

[4] H. Vansompel, P. Sergeant, L. Dupre, and A. van den Bossche, "Axialflux PM machines with variable air gap," IEEE Trans. Ind. Electron., vol. 61, no. 2, pp. 730-737, Feb. 2014.

[5] Y. Gao et al., "Loss reduction of reactor with grain-oriented silicon steel plates," IEEE Trans. Magn., vol. 49, no. 5, pp. 1973-1976, May 2013.

[6] L. R. Dupre, O. Bottauscio, M. Chiampi, M. Repetto, and J. A. A. Melkebeek, "Modeling of electromagnetic phenomena in soft magnetic materials under unidirectional time periodic flux excitations," IEEE Trans. Magn., vol. 35, no. 5, pp. 4171-4184, Sep. 1999.

[7] A. Barchanski, M. Clemens, H. De Gersem, and T. Weiland, "Efficient calculation of current densities in the human body induced by arbitrarily shaped, low-frequency magnetic field sources," J. Comput. Phys., vol. 214, no. 1, pp. 81-95, 2006.

[8] P. Zhou, D. Lin, C. Lu, N. Chen, and M. Rosu, "A new algorithm to consider the effects of core losses on 3-D transient magnetic fields," IEEE Trans. Magn., vol. 50, no. 2, Feb. 2014, Art. ID 7008904.

[9] O. Bíró, K. Preis, and I. Ticar, "A FEM method for eddy current analysis in laminated media," COMPEL, Int. J. Comput. Math. Elect. Electron. Eng., vol. 24, no. 1, pp. 241-248, 2005.

[10] Z. Cheng et al., "Analysis and measurements of iron loss and flux inside silicon steel laminations," IEEE Trans. Magn., vol. 45, no. 3, pp. 1222-1225, Mar. 2009.

[11] R. Stoll, The Analysis of Eddy Currents, 1st ed. Oxford, U.K.: Clarendon, 1974.

[12] G. Bertotti, Hysteresis in Magnetism, 1st ed. New York, NY, USA: Academic, 1998. 